\title{
BP-IL-1
}

\section{Cholangiocarcinoma: Consensus statement from ENS-CCA}

\author{
Jesús María BAÑALES*
}

Biodonostia Health Research Institute, Donostia University Hospital (BHRI-DUH), Donostia-San Sebastian, Spain

Lecture: Cholangiocarcinoma (CCA) includes a heterogenous group of biliary malignant tumours that can arise at any point of the biliary tree. Their incidence is increasing globally, currently accounting for $\sim 15 \%$ of all primary liver cancers and $\sim 3 \%$ of gastrointestinal malignancies. The silent origin and early progression of these tumours combined with their highly aggressive nature and refractoriness to chemotherapy contribute to their alarming mortality, representing $\sim 2 \%$ of all cancer-related deaths worldwide yearly. The current diagnosis of CCA by non-invasive approaches is not accurate enough, and histological confirmation is necessary. Furthermore, the high heterogeneity of CCAs at the genomic, epigenetic and molecular levels severely compromises the efficacy of the available therapies. In the past decade, increasing efforts have been made to understand the complexity of these tumours and to develop new diagnostic tools and therapies that might help to improve patient outcomes. This presentation, based on a recent Expert Consensus Statement [1] endorsed by the European Network for the Study of Cholangiocarcinoma, provides an updated summary and a critical discussion of the latest advances in CCA, mostly focusing on presentation, classification, molecular and genetic abnormalities, biomarkers, management and outcome of patients. Furthermore, the horizon of CCA for the next decade from 2020 onwards is highlighted.

\section{Reference}

1. Banales JM, Marin JJG, Lamarca A, Rodrigues PM, Khan SA, Roberts LR, et al. Cholangiocarcinoma 2020: the next horizon in mechanisms and management. Nat Rev Gastroenterol Hepatol 2020;17:557-588. 